\title{
How Certain Physical Considerations Impact Aerostructural Wing Optimization
}

\author{
John P. Jasa, * Shamsheer S. Chauhan, ${ }^{\dagger}$ \\ University of Michigan, Ann Arbor, MI, USA \\ Justin S. Gray, \\ NASA John H. Glenn Research Center, Cleveland, Ohio \\ Joaquim R. R. A. Martins $§$ \\ University of Michigan, Ann Arbor, MI, USA
}

\begin{abstract}
Wing design optimization has been studied extensively and is of continued interest as optimization tools are developed and become more accessible. In each of these studies, certain assumptions and simplifications are made to make the design problem tractable. However, it is difficult to find systematic studies in which several considerations are added or removed one at a time to study how much impact they have. In this work, we examine how certain physical considerations (viscous drag, wave drag, thrust loads, and inertial relief from structural, fuel, and engine masses), impact the aerostructural optimization results for three distinct aircraft wings. The goal is to help develop a rough idea of how important these physical considerations are. We do this using gradient-based optimization and a multidisciplinary design optimization framework, OpenMDAO. We use the open-source tool OpenAeroStruct that couples a vortex lattice method to a finite element method. We establish a baseline aerostructural design optimization problem then perform a series of optimizations, each with one physical consideration removed from the baseline case. We find that depending on the size of the aircraft and flight conditions, the importance of some of these physical considerations varies considerably whereas the importance of others do not. Specifically, the optimal designs change radically without proper viscous and wave drag considerations and smaller aircraft with more distributed propulsion are more affected by the inclusion of engine loads.
\end{abstract}

\section{Introduction}

Wing aerostructural optimization using numerical methods is of growing interest as the required tools mature and become more accessible [1, 2, 3, 4, 5, 6, 7, 8]. In published studies, different assumptions and simplifications are made to make the design problem tractable. For example, in some aerostructural studies the load inertial relief from engine weights may be included and in some it may not $[3,5]$. Typically, during early-stage design studies, the number of simplifications made are much more numerous than during later stages.

These simplifications are generally made based on the experience and judgment of the designer or researcher, or the limitations of the tools. For someone new to the field of aerostructural design or for someone assessing the limitations of tools, it can be difficult to determine which simplifications are justifiable and how important certain physical considerations are. Additionally, it is difficult to find systematic studies in which several considerations are added or removed one at a time to quantify how important they are.

Preliminary design studies commonly involve using low- to medium-order tools such as vortex-lattice-method (VLM) and finite-element-method (FEM) codes to design wings [9, 10, 4, 11, 12]. Coupling VLM codes for the aerodynamics and FEM codes for the structure allows for rapid optimization studies that consider aerodynamic and

*Ph.D. Candidate, Department of Aerospace Engineering, AIAA Student Member

${ }^{\dagger}$ Ph.D. Candidate, Department of Aerospace Engineering, AIAA Student Member

¥Aerospace Engineer, Propulsion Systems Analysis Branch, 21000 Brookpark Rd., MS 5-11

$\S$ Professor, Department of Aerospace Engineering, AIAA Associate Fellow 
structural trade-offs for a multidisciplinary design optimization (MDO). Even though VLM models and simplified FEM models may not be able to capture as much physics and detail as higher-order methods such as RANS-based computational fluid dynamics (CFD) and detailed FEM models [7, 13], their low cost enables broader design studies that capture major trends with significantly lower computational cost. In this paper, we take advantage of the low cost of VLM and FEM codes to carry out multiple design studies with varying considerations (21 optimization problems in total) to understand different trade-offs. This is useful for understanding how important certain considerations are and which ones must be included in valid design studies.

In this paper we describe how OpenAeroStruct [6], an open-source low-order aerostructural optimization tool that we developed, can be used to understand the impact of different considerations. OpenAeroStruct couples VLM and FEM models together to do aerostructural wing design and is written in Python. The goal of this paper is to help develop a rough idea (involving both qualitative and quantitative intuition) of how important certain physical considerations are compared to others. We carry out series of optimizations for wings based on three significantly different aircraft configurations (long-range transport, regional transport, and short-range commuter) to study how a range of considerations, specifically, viscous drag, wave drag, fuel-weight load relief, structural-weight load relief, engine-weight load relief, and thrust loads from engines impact the optimization results. All of the code used to produce and visualize these results are included in a public open-source repository called Waspy ${ }^{\mathrm{a}}$. We also discuss how OpenMDAO [14], the Python-based multidisciplinary analysis and optimization (MDAO) framework used to build OpenAeroStruct, facilitates creating models with many considerations and sub-disciplines in a modular manner, and setting up varying optimizations studies.

\section{Tools and physics used in this work}

\section{A. OpenMDAO}

MDO is an inherently challenging process because of the complexity of setting up the models and connecting them properly. To perform MDO well, we need a framework that can help facilitate that process of constructing models. Salas and Townsend [15] outlined the basic requirements for an MDO application framework. Padula and Gillian [16] surveyed the field at the time, detailed the history of MDO frameworks, and found there were four key concepts among the frameworks: modularity, data handling, parallel processing, and user interface. These concepts are prevalent among existing MDO frameworks, such as RCE [17], SORCER [18], Spiral [19], ModeFrontier, ModelCenter, and OpenMDAO [14]. These frameworks each have their own advantages and common application cases. They might have graphical user interfaces (GUIs) to ease model creation, derivative-handling to enable gradient-based optimization, the ability to couple external codes, or distributed computing capabilities.

We will not compare MDO frameworks here. Instead, we will focus on one — NASA's OpenMDAO [14] — and showcase its ability to enable gradient-based multidisciplinary optimization in a modular manner. OpenMDAO solves coupled systems using Newton-type algorithms and provides a framework to facilitate derivative computation [14]. Additionally, models can be constructed in a modular manner with any user-defined hierarchy of solvers.

Throughout this work, we use OpenMDAO [14] as the underlying optimization framework. OpenMDAO was developed at NASA Glenn and uses the modular analysis and unified derivatives theory to allow for modular construction and execution of complicated models [20]. OpenMDAO has been used to optimize a variety of problems, including wind turbines [21, 22], boundary layer ingestion aircraft [23, 24], thermodynamic engine cycles [25, 26], and coupled thermal-mission problems [6, 27].

OpenMDAO was designed from the ground-up with gradient-based optimization in mind. Because of this, a lot of the challenging aspects associated with propagating derivatives through a complicated model are handled behindthe-scenes by OpenMDAO without user input. Specifically, if a user is creating a model that consists of multiple analysis blocks, or components, and wants to connect them into a group to perform gradient-based optimization, the user only needs to provide partial derivatives for the individual analysis blocks, not total derivatives for the entire model. OpenMDAO uses the chain rule to compute the total derivatives based on the partial derivatives computed from each analysis block. Additionally, if the user is prototyping a new model or does not want to provide derivatives, they can tell OpenMDAO to use finite-difference or complex-step [28] approximations to obtain the derivatives. This

\footnotetext{
${ }^{a}$ https://github.com/johnjasa/waspy
} 
kind of heterogeneous model and derivative construction allows users to create complicated models that are suitable for gradient-based optimization relatively quickly.

For the work presented in this paper, we are interested in studying the effects of different physical considerations on optimal wing design. Given a monolithic model, it would be challenging to set up gradient analysis for all permutations of the physics considered. However, we instead create individual modular components and groups and connect them differently based on the problem we are modeling. Because OpenMDAO handles the data- and derivative-passing internally once we issue data connections, we can straightforwardly alter the aerostructural wing model.

\section{B. OpenAeroStruct}

We will examine and document the capabilities of OpenMDAO using a specific existing model, OpenAeroStruct [6]. OpenAeroStruct is an open-source design tool that couples a vortex lattice method (VLM) with a 6-degree-of-freedom finite element model to perform aerostructural analysis and optimization. It has been used for a wide variety of studies, including morphing aircraft trajectory optimization [29], uncertainty quantification on performance of unmanned aircraft [30], comparisons to high-fidelity aerostructural optimization results [7], and design of electric aircraft [31]. OpenAeroStruct is written using only Python within the OpenMDAO framework and is useful to students, educators, and researchers.

For this work, we use aerostructural optimization problems implemented in OpenAeroStruct to conduct our wing design studies. An extended design structure matrix (XDSM) [32] of a simple aerostructural wing design problem is shown in Fig. 1. The optimizer supplies design variable values (twist and thickness distributions) to the analyses. First, geometric properties of the wing are computed, then the aerodynamic and structural analyses are converged using a solver, and the converged aerostructural solution is passed onto postprocessing analyses. OpenAeroStruct is capable of running both analysis and optimization, though in this paper we focus on results from converged optimization cases. The actual optimization formulation used in these studies is slightly more complex than what is shown in Fig. 1, which has been simplified for clarity.

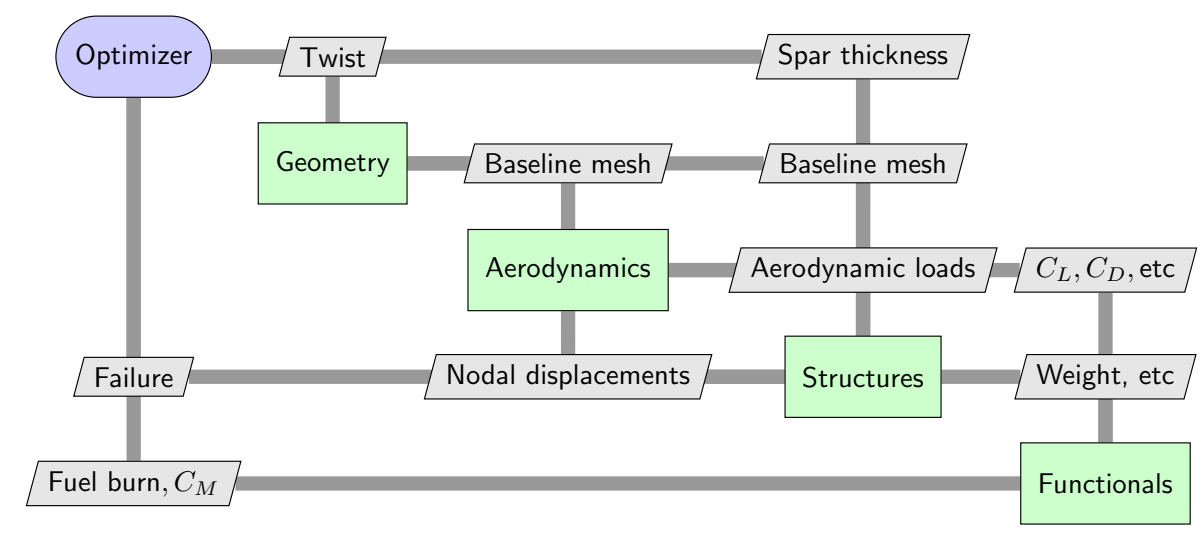

Figure 1: Extended design structure matrix [32] of an OpenAeroStruct model

Nominally, an OpenAeroStruct model consists of a collection of components and groups that perform the aerostructural analysis and process the results. Users can expand these models by creating and connecting other analyses within the OpenMDAO framework. This means that OpenAeroStruct models can easily be integrated into larger-scale optimization problems considering more disciplines, or the base models can be trimmed down to just the relevant analyses for the current study. Additionally, users can set the solver hierarchy and Jacobian construction methods at the run script level, which is a feature of OpenMDAO's modularity. 


\section{Explanation of existing model features}

Prior to the work presented in this paper, a variety of physical considerations had already been implemented in OpenAeroStruct. Before detailing what has been added as a result of this work, we first summarize the existing features.

\section{Viscous drag}

To supplement the induced drag computed using the VLM model, OpenAeroStruct includes viscous-drag estimates computed using skin-friction-coefficient and form-factor formulas from Raymer [33] (specifically Sec. 12.5.3 and Sec. 12.5.4 of the 5th edition). Bons et. al. [34, 35] implemented this and studied how the inclusion of viscous-drag terms changed wing aerodynamic optimization results.

\section{Wingbox model}

In the first iteration of OpenAeroStruct, the cross-sectional properties of the structural model were computed assuming a tubular-spar internal structure for computational simplicity [6]. This is valid for small RC-scale aircraft and some stunt planes, but not for the majority of transport aircraft. Chauhan and Martins [7] implemented a wingbox model for a more accurate representation of typical transport-aircraft wing structures and found that the optimized fuel-burn and structural-weight results matched high-fidelity results [13] within 10\% for the undeflected Common Research Model (uCRM-9 [5]) wing.

To use this model, the user provides airfoil-shape coordinates for the wingbox cross-section and chooses how much of the chord is occupied by the wingbox. The skin and spar thicknesses can be provided as fixed inputs or controlled as optimization design variables. A cross-sectional view of the wingbox representation adapted from Chauhan and Martins [7] is shown in Fig. 2. Thickness-to-chord ratio design variables can also be used scale the wingbox crosssections.

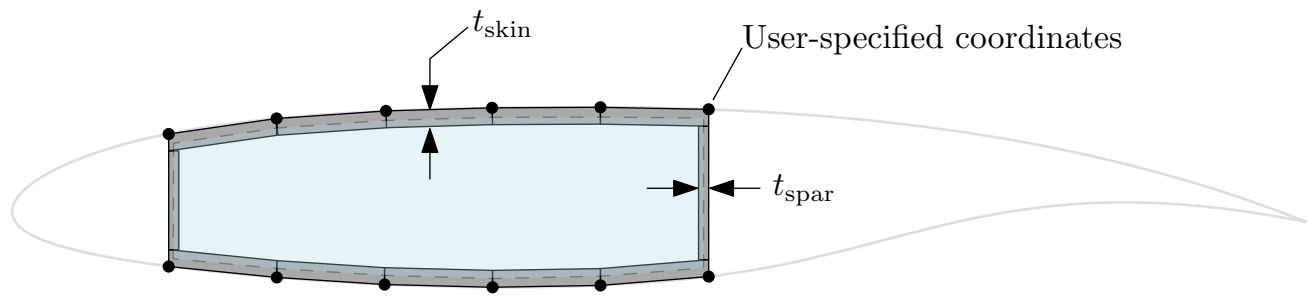

Figure 2: Cross-sectional view of the wingbox model with the spar and skin thicknesses labeled [7]

A planform view of an FEM mesh superimposed on a VLM mesh is shown in Fig. 3. The FEM model uses the spanwise spacing of the VLM mesh for the spatial beam elements. The wingbox model is described in more detail by Chauhan and Martins [7].

\section{Wave drag}

To account for the wave drag of the wing, which is important in transonic flight conditions, Chauhan and Martins [7] also implemented an estimate using the following equations based on the Korn equation [36]:

$$
M_{\text {crit }}=\frac{\kappa}{\cos \Lambda}-\frac{t / c}{\cos ^{2} \Lambda}-\frac{C_{\mathrm{L}}}{10 \cos ^{3} \Lambda}-\left(\frac{0.1}{80}\right)^{1 / 3}
$$

and

$$
C_{\mathrm{D}, \text { wave }}=20\left(M-M_{\text {crit }}\right)^{4} .
$$

Here, $M$ is the flight Mach number, $M_{\text {crit }}$ is the critical Mach number, $\kappa$ is an airfoil-technology factor (set to 0.95 for NASA supercritical airfoils), $t / c$ is the streamwise thickness-to-chord ratio, $C_{\mathrm{L}}$ is the wing coefficient of lift, and $\Lambda$ 


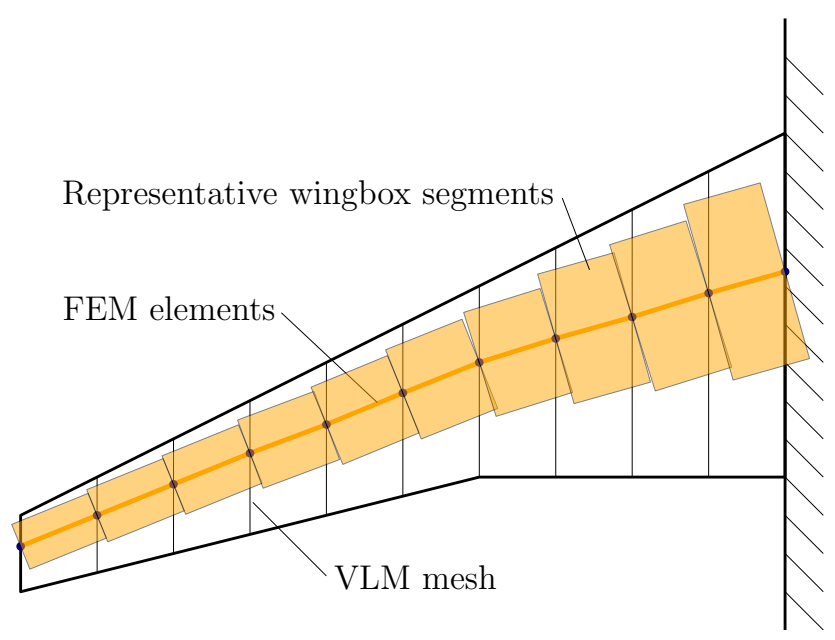

Figure 3: A planform view of an illustrative wing mesh showing the VLM mesh and the FEM mesh with representative wingbox segments [7]

is the sweep angle. The cosine of the sweep angle, $\cos \Lambda$, is computed by averaging the cosines of the quarter-chord sweep angles for each spanwise segment of the VLM mesh, weighted by their areas. Similarly, the average thicknessto-chord ratio, $t / c$, is computed by averaging the thickness-to-chord ratios corresponding to each spanwise segment, weighted by their areas [7].

\section{Structural-weight and fuel-weight load relief}

Chauhan and Martins [7] also added features to allow applying distributed loads on the wing for the weight of the structure itself, as well as the weight of the fuel stored in the wings [7]. These considerations add forces on the wing which counteract the upward lift forces, reducing shear loads and bending moments on the structure during positive load-factor flight. Additionally, the internal volume of the wingbox can be used to add a fuel-in-wing volume constraint to ensure that sufficient volume is available to store the required fuel in the wings.

\section{Model features added for this work}

For this current work, we added the ability to add point loads to the aerostructural wing model. We are primarily interested in considering loads due to the weight of engines and loads due to the thrust from the engines. However, these point loads can be used to represent the weights of other components such as control surfaces and actuators, batteries, and armaments. Previous high-fidelity aerostructural wing design studies have considered engine masses and control surface actuator masses [5].

We compute the forces and moments acting on the FEM nodes, due to the point loads, based on the inverse spanwise distance between them and the location of the point loads raised to the tenth power. On many transport aircraft, an engine is supported by a pylon that connects to a portion of the wing structure, transferring the loads to multiple attachment points. However, we do not model the pylon connection. Instead we opt for an inverse spanwise distance approach so that we can consider optimization problems in which the wing mesh can move relative to the locations of the point loads (e.g., if we have span as a design variables). This allows smooth gradients for gradientbased optimization. This also allows the user to easily change mesh resolution without re-selecting which nodes to transfer loads to, and re-calculating what fraction of the loads to apply to each node. The sum of the forces applied to the wing add up to the magnitude of the specified point force. However, this method is not guaranteed to be work conservative. The nodes closest to the point mass bear the majority of the loading, and the amount applied to other nodes decreases drastically away from them due to the large exponent on the inverse distance. 
These additions to the OpenAeroStruct model were straightforward to implement and integrate into the existing codebase due to the modular formulation afforded through the use of OpenMDAO. Without this MDO framework, implementing efficient derivatives quickly as we updated the model would be much more challenging, as we might need to structure other portions of the code. Instead, we only needed to provide the new components and connect them into the existing model, and through the chain rule we are able to obtain low-cost total derivatives for use in gradient-based optimization. OpenMDAO also allows us to not supply analytic gradients and instead compute the partial derivatives using finite-difference or complex-step methods [28], which makes the prototyping process easier and quicker.

\section{Wing models and case definition}

We now define the physical properties of the three aircraft wings we study here. Because we are interested in studying the effects that different physical considerations have on optimal wing design in general, we examine three distinct wings based on long-range, regional, and short-range commercial transport aircraft. We examine a wing based on the uCRM-9 (similar in size to a Boeing 777) [5], a wing based on the Bombardier Q400, and a wing based on a 15passenger short-range commuter concept developed by NASA researchers [37]. The planform views of the half-wing meshes for these three aircraft are shown using the same scale in Fig. 4.

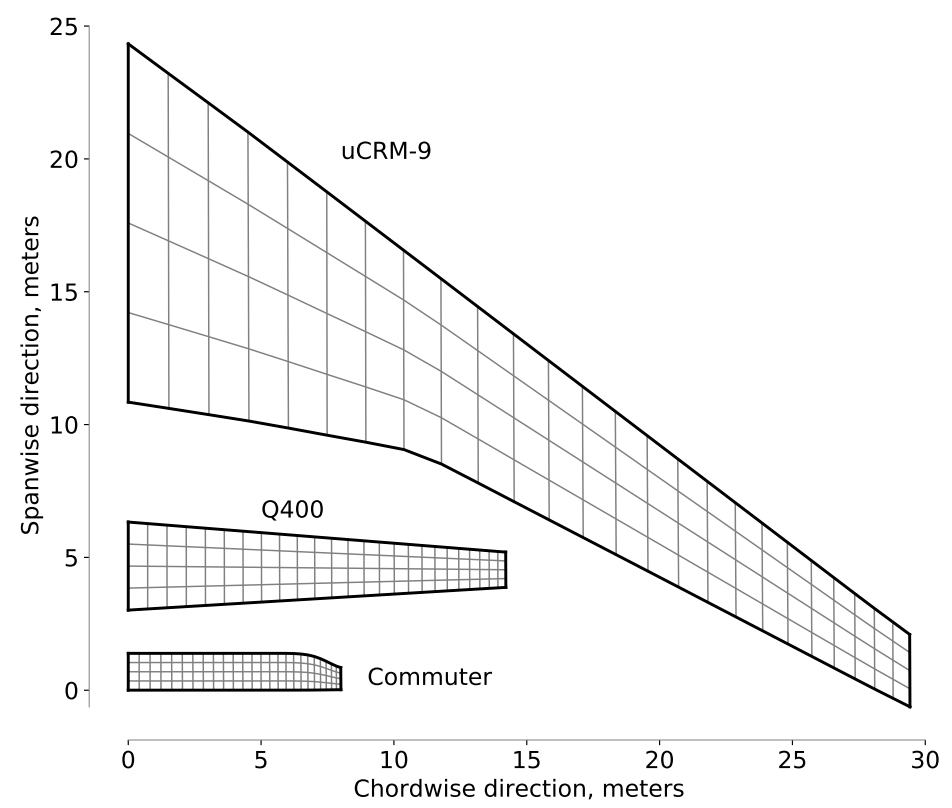

Figure 4: To-scale planform views of the three wings (half-span) used in this paper with their VLM meshes overlaid

\section{A. Wing properties and definition}

We use the uCRM-9 geometry [13,5] for the long-range transport wing. The uCRM-9 is based on the CRM [38], but modified to remove the flying-shape deflections. The CRM aircraft is similar in size and performance to a Boeing 777.

For the regional-aircraft wing, we use specifications of the Bombardier Q400 Dash $8^{\mathrm{b}}$. The Q400 Dash 8 is powered by two propellers, carries up to 90 passengers, has a maximum takeoff weight (MTOW) of 30,500 kg, and

\footnotetext{
b Q400 factsheet https://commercialaircraft.bombardier.com/themes/bca/pdf/Factsheet_Q_Series_Q400_2018.pdf [Accessed: May 2019]
} 
cruises at around Mach 0.50. We used images from the Q400 Airport Planning Manual ${ }^{\mathrm{c}}$ to create the wing planform mesh.

Our third wing is based on a new NASA tiltwing concept detailed by Johnson et. al. [37]. This concept is designed for urban-air-mobility applications. Although this aircraft's wing tilts up for vertical takeoff and landing, it cruises in a conventional configuration. For this paper, we do not consider the tilting ability of the wing. This aircraft is a short-range commuter that carries 15 passengers, has an MTOW of $6500 \mathrm{~kg}$, and cruises around Mach 0.30.

The parameters and specifications used in these studies for all three of these wings are listed in Table 1. The 'wing mass factor' is a multiplicative factor on the computed wingbox mass to account for other masses in the wing that are not modeled, such as fasteners and overlaps [5] or leading and trailing edge weights. When we discuss 'wing mass' in the rest of the paper, those values include the multiplicative factor.

Table 1: Specifications and parameter estimates

\begin{tabular}{|c|c|c|c|c|c|}
\hline \multirow[b]{2}{*}{ Parameter } & \multicolumn{3}{|c|}{ Value } & \multirow[b]{2}{*}{ Units } & \multirow[b]{2}{*}{ Comments } \\
\hline & uCRM-9 & Q400 & Commuter & & \\
\hline Wing span & $58.8[5]$ & $28.4^{\mathrm{b}}$ & $16.0[37]$ & $\mathrm{m}$ & \\
\hline Centerline chord & $13.6[5]$ & $3.3^{\mathrm{c}}$ & $1.4[37]$ & $\mathrm{m}$ & \\
\hline Tip chord & $2.7[5]$ & $1.3^{\mathrm{c}}$ & $0.8[37]$ & $\mathrm{m}$ & \\
\hline Wingbox elastic modulus, $E$ & 73.1 & 73.1 & 73.1 & $\mathrm{GPa}$ & Al 7000 series alloy [5] \\
\hline Wingbox Poisson's ratio, $v$ & 0.3 & 0.3 & 0.3 & - & Al 7000 series alloy [5] \\
\hline Wingbox shear modulus, $G$ & 28.1 & 28.1 & 28.1 & $\mathrm{GPa}$ & $G=\frac{E}{2(1+v)}$ \\
\hline Wingbox yield strength & 420 & 420 & 420 & $\mathrm{MPa}$ & Al 7000 series alloy [5] \\
\hline Wingbox density & 2780 & 2780 & 2780 & $\mathrm{~kg} / \mathrm{m}^{3}$ & Al 7000 series alloy [5] \\
\hline Portion of chord occupied by wingbox & $10 \%$ to $60 \%$ & $10 \%$ to $60 \%$ & $10 \%$ to $60 \%$ & - & Assumed based on Refs. [5, 7] \\
\hline Yield-stress safety factor & 1.5 & 1.5 & 1.5 & - & Assumed based on Ref. [5] \\
\hline Wing mass factor & $1.25[5]$ & 1.25 & 2.0 & - & Assumed \\
\hline Rest-of-aircraft $C_{\mathrm{D} 0}$ & $0.0078[7]$ & $0.0142[39]$ & 0.0110 & - & Component buildup [33] and assumed \\
\hline Thrust-specific fuel consumption (TSFC) & $0.53[5]$ & 0.43 & 0.45 & $\mathrm{lb} /(\mathrm{lbf} \cdot \mathrm{hr})$ & Assumed (for cruise) \\
\hline Per-engine mass & $7500[5]$ & 1050 & 175 & $\mathrm{~kg}$ & Assumed \\
\hline Per-engine cruise thrust & 80,000 & 8000 & 250 & $\mathrm{~N}$ & Assumed \\
\hline Total number of engines on aircraft & 2 & 2 & 4 & - & \\
\hline Mission range & $14,300[5]$ & $2000^{\mathrm{b}}$ & $740[37]$ & $\mathrm{km}$ & \\
\hline Zero-fuel weight without wing mass & $148,000[5]$ & 25,400 & 6000 & $\mathrm{~kg}$ & Assumed \\
\hline Fuel density & 803 & 803 & 803 & $\mathrm{~kg} / \mathrm{m}^{3}$ & Jet A-1 [5] \\
\hline Reserve fuel & $15,000[5]$ & 500 & 150 & $\mathrm{~kg}$ & Assumed \\
\hline Cruise Mach number & $0.85[5]$ & $0.50^{\mathrm{b}}$ & $0.30[37]$ & - & \\
\hline Cruise altitude & $37,000[5]$ & $24,000^{\mathrm{b}}$ & $5000[37]$ & $\mathrm{ft}$ & \\
\hline Maneuver Mach number & $0.64[5]$ & 0.30 & 0.225 & - & Assumed \\
\hline Maneuver altitude & $0[5]$ & 0 & 0 & $\mathrm{ft}$ & Assumed \\
\hline
\end{tabular}

\section{Optimization problem formulations}

We now detail the optimization problem formulations used in each of the comparative studies. We first present a baseline case with all of our physical considerations included, then create a series of optimization cases, each with one physical consideration removed from the baseline case. Table 2 explicitly lists the cases and physics considered for each case.

Most of the physical considerations listed in Table 2 are included in published studies, especially viscous and wave drag, structural- and fuel-weight relief, and engine-weight relief. However, engine-thrust loads are not incorporated

${ }^{c}$ Estimated from the Q400 Airport Planning Manual PSM 1-84-13 https://customer.aero.bombardier.com/webd/BAG/CustSite/ BRAD/RACSDocument.nsf/51aae8b2b3bf df6685256c300045ff31/ec63f8639ff3ab9d85257c1500635bd8/\$FILE/ATTNBE0B . pdf/ D8400-APM.pdf [Accessed: May 2019] 
in most wing design studies, [5, 40], but has been considered in other work that had detailed structural load considerations [41]. Through these studies, we can quantify the effect that each of these considerations have on the optimal wing design for a range of aircraft sizes.

Table 2: Cases and physics considered where $\checkmark$ means that case included that consideration

Physics considered

\begin{tabular}{lcccccc} 
Case name & Viscous drag & Wave drag & Struct-weight relief & Fuel-weight relief & Engine-weight relief & Engine thrust \\
\hline Baseline & $\checkmark$ & $\checkmark$ & $\checkmark$ & $\checkmark$ & $\checkmark$ & $\checkmark$ \\
w/o viscous drag & & $\checkmark$ & $\checkmark$ & $\checkmark$ & $\checkmark$ & $\checkmark$ \\
w/o wave drag & $\checkmark$ & & $\checkmark$ & $\checkmark$ & $\checkmark$ & $\checkmark$ \\
w/o struct-weight relief & $\checkmark$ & $\checkmark$ & $\checkmark$ & $\checkmark$ & $\checkmark$ \\
w/o fuel-weight relief & $\checkmark$ & $\checkmark$ & $\checkmark$ & $\checkmark$ & $\checkmark$ \\
w/o engine-weight relief & $\checkmark$ & $\checkmark$ & $\checkmark$ & $\checkmark$ & \\
w/o engine thrust & $\checkmark$ & $\checkmark$ & & & $\checkmark$ \\
\hline
\end{tabular}

Table 3 summarizes the baseline optimization problem. We allow the optimizer to control the twist distribution, spar-thickness distribution (same for the front and rear spars), skin-thickness distribution (same for the upper and lower skins), and thickness-to-chord ratio distributions, to minimize fuel burn subject to a set of constraints. These distributions are parameterized using B-splines. This benchmark problem considers a multipoint formulation in which the fuel burn is computed at a nominal cruise lift coefficient, and $2.5 \mathrm{~g}$ maneuver loads are used to size the wing structure. We also allow the optimizer to vary the angle of attack for the $2.5 \mathrm{~g}$ maneuver case to ensure that the wing produces sufficient lift. We include lift-equals-weight, $C_{\mathrm{L}}$, structural failure, and fuel-in-wing volume constraints. This problem has been studied before using OpenAeroStruct by Chauhan and Martins [7].

The fuel burn, which is the objective function for each of the optimization problems, is computed using the Breguet range equation. The following is the equation for fuel burn obtained by rearranging the Breguet range equation:

$$
W_{\mathrm{f}}=\left(W_{0}+W_{\mathrm{s}}\right)\left[\exp \left(\frac{R \cdot \mathrm{TSFC}}{V}\left(\frac{L}{D}\right)^{-1}\right)-1\right] \text {, }
$$

where $W_{\mathrm{f}}$ is the fuel weight, $W_{0}$ is the aircraft empty weight, $W_{\mathrm{s}}$ is the structural weight, $R$ is the range, $V$ is the speed, $L / D$ is the lift-to-drag ratio, and TSFC is the thrust-specific fuel consumption.

Table 3: Nominal formulation for the aerostructural optimization problems. The short-range commuter has thicknesses lower bounds of $0.001 \mathrm{~m}$ and $C_{\mathrm{L} \text {,cruise }}=0.6$ for the $\mathrm{Q} 400$.

\begin{tabular}{llrrrr}
\hline Category & Name & Lower & Upper & Units & Quantity \\
\hline Objective function & fuel burn & & & $\mathrm{kg}$ & 1 \\
\hline Design variables & wing twist & -15 & 15 & degrees & 6 \\
& thickness-to-chord ratio & 0.06 & 0.20 & & 6 \\
& spar thickness & 0.003 & 0.5 & $\mathrm{~m}$ & 6 \\
& skin thickness & 0.003 & 0.5 & $\mathrm{~m}$ & 6 \\
& $\alpha_{2.5 \mathrm{~g}}$ & -15 & 15 & degrees & 1 \\
& Total & & & & $\mathbf{2 5}$ \\
\hline Constraints & $C_{\mathrm{L}, \text { cruise }}$ & 0.5 & 0.5 & & 1 \\
& lift $2.5 \mathrm{~g}$ & weight $_{2.5 \mathrm{~g}}$ & weight $_{2.5 \mathrm{~g}}$ & $\mathrm{~N}$ & 1 \\
& $\sigma_{\text {von Mises, } 2.5 \mathrm{~g}}$ & & 280 & $\mathrm{MPa}$ & 1 \\
& fuel volume & & wingbox volume & $\mathrm{m}^{3}$ & 1 \\
& Total & & & & $\mathbf{4}$ \\
\hline
\end{tabular}


For all optimization cases presented here, we used a gradient-based optimizer, SNOPT [42], through the opensource Python interface pyOptSparse ${ }^{\mathrm{d}}$ [43]. All optimization cases were converged below $10^{-7}$ optimality and feasibility tolerances. Because SNOPT is not freely available to the public, the cases included in the Waspy repository use Scipy's SLSQP [44] method.

Figure 5 shows the optimized skin-thickness distributions for the uCRM-9 wing from OpenAeroStruct compared to the results from high-fidelity (RANS CFD and shell-element FEM) optimization [13]. We see reasonable agreement in the outboard part of the wing, but there are significant differences at the root. One factor that contributes to this is that the high-fidelity model had constraints limiting displacements at the fuselage junction [13], something that we do not model with OpenAeroStruct. The two models differ significantly in a few other ways as well, apart from just the aerodynamics and structural tools used. The high-fidelity model included the fuselage, the horizontal tail, buckling constraints, and sizing for skin stiffeners, and also allowed for different upper and lower skin thicknesses, whereas the OpenAeroStruct model is wing-only, has no buckling constraints or stiffeners, and uses the same values of skin thickness for the upper and lower skins. The high-fidelity optimization problem also had additional load cases $(--1 \mathrm{~g}$ maneuver and cruise with gust cases) and buffet constraints. The OpenAeroStruct optimization took tens of minutes to converge on a desktop and the high-fidelity case took 48 hours on 1000 processors [13].

Figure 5 also compares the optimized thickness-to-chord ratios. We see that although the ranges are similar, the trends do not match. OpenAeroStruct uses empirical viscous and wave-drag formulas (described in Sec. C), whereas the high-fidelity model captures the flow physics around the wing more accurately. This difference partially accounts for the difference in thickness-to-chord ratio trends. Additionally the high-fidelity optimization also includes the fuselage and interacting wing-body aerodynamics as well as hundreds of airfoil shape variables [13].

\footnotetext{
$\mathrm{d}_{\text {https://github.com/mdolab/pyoptsparse }}$
} 


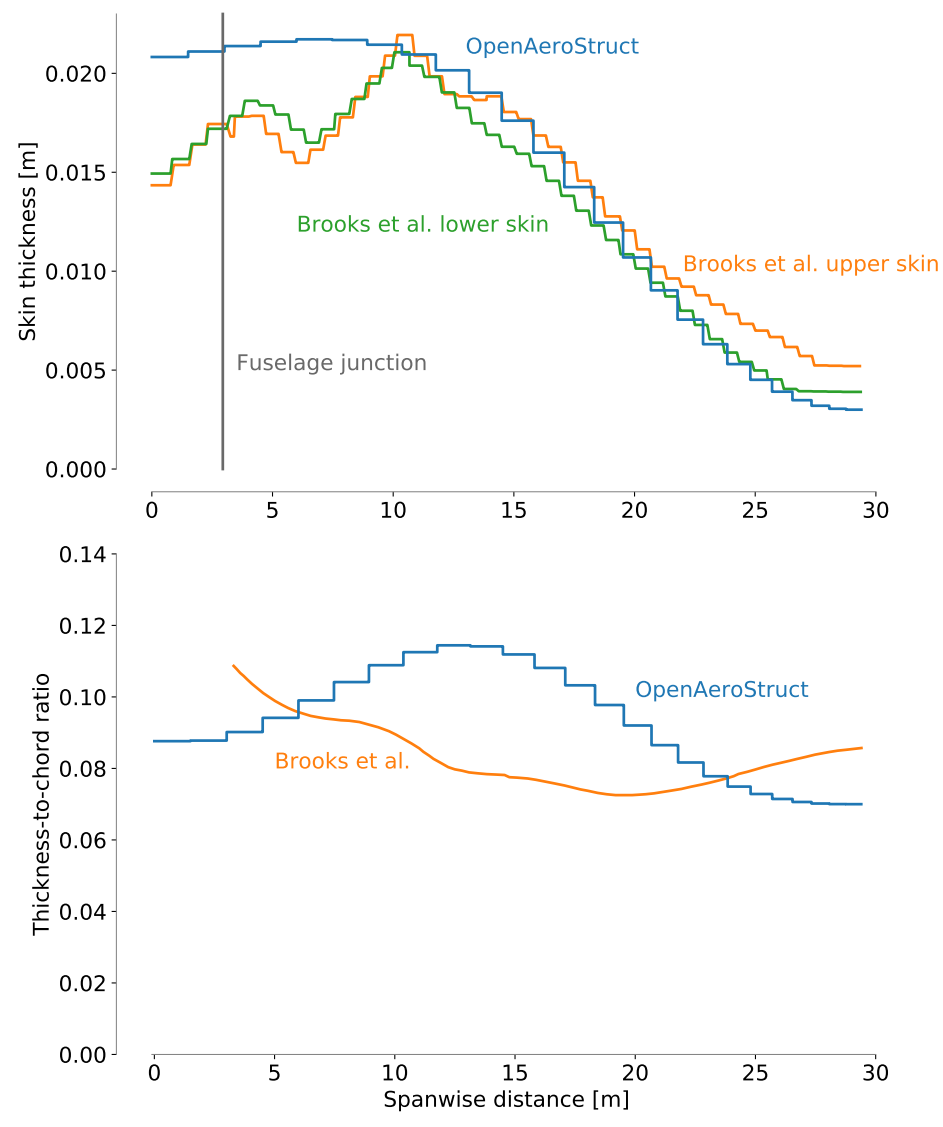

Figure 5: High-fidelity results of Brooks et al. [13] and OpenAeroStruct results shows reasonable agreement for the optimized skin thickness for the uCRM-9 wing.

\section{Discussion of optimization results}

We now interpret the results from each of the 21 optimization cases. Through these comparisons, we can see how much of an effect each of these physical considerations has on the optimization results. Nominally, we can make educated guesses about some trends we would see based on the wing size and flight conditions, but through these results we can quantify the changes in design and performance. Tables 4,5 , and 6 show the optimized performance metrics for each wing, as well as the percentage change from the baseline optimization results for each case.

Figure 6 shows the optimal skin thickness and thickness-to-chord ratio distributions for all seven cases for each of the three aircraft and Fig. 7 shows the optimal lift and twist distributions. In each of the figures, some distributions are plotted as steps and others are continuous. For the skin-thickness and thickness-to-chord step distributions, the aerostructural model uses the same constant value across the given structural element and chordwise aerodynamic panel. The continuous distributions for twist show the continuous B-spline representation of the design variables. 
Table 4: Optimized performance metrics for the uCRM-9

\begin{tabular}{|c|c|c|c|c|c|c|c|c|}
\hline & \multicolumn{2}{|c|}{ Fuel burn } & \multicolumn{2}{|c|}{ Wing mass } & \multicolumn{2}{|c|}{ Drag } & \multicolumn{2}{|c|}{ MTOW } \\
\hline & $\mathrm{kg}$ & $\Delta, \%$ & $\mathrm{~kg}$ & $\Delta, \%$ & counts & $\Delta, \%$ & $\mathrm{~kg}$ & $\Delta, \%$ \\
\hline Baseline & $93,825.6$ & 0.0 & $26,292.7$ & 0.0 & 239.7 & 0.0 & $283,021.6$ & 0.0 \\
\hline w/o viscous drag & $60,917.5$ & -35.1 & $24,599.0$ & -6.4 & 167.4 & -30.1 & $248,431.6$ & -12.2 \\
\hline w/o wave drag & $91,027.6$ & -3.0 & $18,408.2$ & -30.0 & 242.1 & 1.0 & $272,342.8$ & -3.8 \\
\hline w/o struct-weight relief & $95,252.7$ & 1.5 & $27,906.2$ & 6.1 & 241.0 & 0.5 & $286,061.2$ & 1.1 \\
\hline w/o fuel-weight relief & $98,637.9$ & 5.1 & $34,210.5$ & 30.1 & 241.5 & 0.7 & $295,747.4$ & 4.5 \\
\hline w/o engine-weight relief & $94,248.1$ & 0.5 & $27,071.5$ & 3.0 & 239.8 & 0.0 & $284,222.5$ & 0.4 \\
\hline w/o engine thrust & $93,837.7$ & 0.0 & $26,275.7$ & -0.1 & 239.7 & 0.0 & $283,016.8$ & -0.0 \\
\hline
\end{tabular}

Table 5: Optimized performance metrics for the Q400

\begin{tabular}{|c|c|c|c|c|c|c|c|c|}
\hline & \multicolumn{2}{|c|}{ Fuel burn } & \multicolumn{2}{|c|}{ Wing mass } & \multicolumn{2}{|c|}{ Drag } & \multicolumn{2}{|c|}{ MTOW } \\
\hline & $\mathrm{kg}$ & $\Delta, \%$ & $\mathrm{~kg}$ & $\Delta, \%$ & counts & $\Delta, \%$ & $\mathrm{~kg}$ & $\Delta, \%$ \\
\hline Baseline & 2303.4 & 0.0 & 1889.1 & 0.0 & 311.0 & 0.0 & $30,082.2$ & 0.0 \\
\hline w/o viscous drag & 1685.6 & -26.8 & 1454.7 & -23.0 & 233.5 & -24.9 & $29,030.3$ & -3.5 \\
\hline w/o wave drag & 2303.4 & 0.0 & 1889.1 & 0.0 & 311.0 & 0.0 & $30,082.2$ & 0.0 \\
\hline w/o struct-weight relief & 2309.9 & 0.3 & 1925.5 & 1.9 & 311.4 & 0.1 & $30,125.1$ & 0.1 \\
\hline w/o fuel-weight relief & 2312.2 & 0.4 & 1954.5 & 3.5 & 311.4 & 0.1 & $30,156.4$ & 0.2 \\
\hline w/o engine-weight relief & 2307.0 & 0.2 & 1916.1 & 1.4 & 311.1 & 0.1 & $30,112.8$ & 0.1 \\
\hline w/o engine thrust & 2302.8 & -0.0 & 1882.6 & -0.3 & 311.0 & -0.0 & $30,075.2$ & -0.0 \\
\hline
\end{tabular}

Table 6: Optimized performance metrics for the short-range commuter

\begin{tabular}{|c|c|c|c|c|c|c|c|c|}
\hline & \multicolumn{2}{|c|}{ Fuel burn } & \multicolumn{2}{|c|}{ Wing mass } & \multicolumn{2}{|c|}{ Drag } & \multicolumn{2}{|c|}{ MTOW } \\
\hline & $\mathrm{kg}$ & $\Delta, \%$ & $\mathrm{~kg}$ & $\Delta, \%$ & counts & $\Delta, \%$ & $\mathrm{~kg}$ & $\Delta, \%$ \\
\hline Baseline & 313.5 & 0.0 & 498.4 & 0.0 & 254.1 & 0.0 & 6959.5 & 0.0 \\
\hline w/o viscous drag & 209.7 & -33.1 & 366.5 & -26.5 & 174.6 & -31.3 & 6723.9 & -3.4 \\
\hline w/o wave drag & 313.5 & 0.0 & 498.4 & 0.0 & 254.1 & 0.0 & 6959.5 & 0.0 \\
\hline w/o struct-weight relief & 314.5 & 0.3 & 507.4 & 1.8 & 254.5 & 0.2 & 6969.6 & 0.1 \\
\hline w/o fuel-weight relief & 314.9 & 0.5 & 514.6 & 3.2 & 254.6 & 0.2 & 6977.1 & 0.3 \\
\hline w/o engine-weight relief & 316.9 & 1.1 & 544.5 & 9.3 & 255.0 & 0.4 & 7009.0 & 0.7 \\
\hline w/o engine thrust & 313.4 & -0.0 & 499.6 & 0.2 & 253.9 & -0.0 & 6960.7 & 0.0 \\
\hline
\end{tabular}

\section{A. Impact on wing mass}

Before discussing the impact on the wing mass, we use the term 'wing mass' to mean the mass of the wingbox structure multiplied by the wing mass factor (listed in Table 1). Starting with the uCRM-9, we see that the fuel-weight load relief and wave-drag considerations have the largest impact on the skin thickness distributions (Fig. 6a) and wing mass (30\% increase w/o fuel-weight relief and 30\% decrease w/o wave drag). The structural-weight load relief and viscous-drag considerations do not have as large an impact and change the wing mass by less than $10 \%$. Neglecting the structural-weight relief reduced the wing mass by $6 \%$ and neglecting the viscous drag increased the wing mass by $6 \%$. The impacts of the engine-weight and the engine-thrust considerations are the lowest. The engine-weight load relief reduced the wing mass by $3 \%$ and the engine thrust had a negligible impact on the wing mass. This shows that for long-range transport aircraft, which usually operate in the transonic regime, ensuring that wave-drag and fuelweight relief considerations are taken into account is important for preliminary structural sizing. Accounting for the 
engine-weight relief and thrust loads are the least important and can be expected to impact the wing mass by only a few percent.

For the Q400, unlike the uCRM-9, the wave-drag and fuel-weight relief considerations do not change the skin thickness distributions (Fig. 6c) and wing mass by a large margin. This is not surprising because the Q400 does not operate in the transonic regime and because the fuel weight for the Q400 (a regional aircraft) is a much smaller fraction of the MTOW compared to the uCRM-9 (a long-range aircraft). Neglecting wave drag had a negligible impact on the wing mass and neglecting fuel-weight relief increased the wing mass by only a few percent. Also, in contract with the uCRM-9 results, neglecting viscous drag has the largest impact on the wing mass. Without being penalized by wave drag, the thickness-to-chord ratio of the wing can increase significantly to reduce the required structural mass when viscous drag is neglected. The rest of the considerations impact the wing mass by only a few percent. This shows that for regional aircraft that operate in the mid-subsonic regime, ensuring that wave-drag, fuel-weight relief, structural-weight relief, and engine-weight relief considerations are taken into account is not important for preliminary structural sizing. These considerations impact the wing mass by a few percent or less.

For the short-range commuter, as with the Q400, neglecting viscous drag has the largest impact on the wing mass. However, the engine-weight relief has a larger impact on the wing mass than for the Q400 (9\% compared to 1\%). This is not surprising due to the locations of the engines. The results shows that for short-range commuter turboprops that operate in the subsonic regime, ensuring that fuel-weight relief and structural-weight relief considerations are taken into account is not important for preliminary structural sizing. These considerations can be expected to impact the wing mass by only a few percent. However, with the propulsion distributed on the wing, considering the load relief from the engines (or motors) can be expected to impact the wing mass in the order of $10 \%$.

\section{B. Impact on fuel burn}

For all the cases, neglecting the viscous drag for the wing has a large impact on the fuel-burn estimates (around 30\%). This is not surprising and is the reason why it is uncommon to neglect viscous drag when estimating fuel burn at any stage of the design process. For the uCRM-9, all the other considerations impacted the fuel burn by $5 \%$ or less, with the fuel-weight relief consideration having the greatest impact. For the Q400, all the other considerations impacted the fuel burn by less than $1 \%$. For the short-range commuter, all the other considerations impacted the fuel burn by $1 \%$ or less. These results show that fuel-burn estimates for long-range missions are more significantly impacted by load relief considerations. However, the impact is only a few percent.

\section{Impact on optimal designs}

Figure 6 shows that the skin thickness distributions vary the most in the uCRM-9 cases, whereas the Q400 and commuter thickness distributions are not as significantly affected by the different physical considerations. For the uCRM- 9 case, fuel-weight relief, structural-weight relief, viscous drag, and wave drag all significantly change the optimal thickness distribution, but only viscous drag greatly impacts the thickness distributions for the other two cases.

The thickness-to-chord ratio affects the wave-drag estimates, viscous-drag estimates, and heights of the wingbox segments. Figure $6 \mathrm{~b}$ shows that the wave-drag, fuel-weight relief, and structural-weight relief considerations all significantly impact the optimal thickness-to-chord ratio distribution for the uCRM-9 case. Specifically, without wave drag, the optimal design is noticeably thicker across the entire span, and without fuel-weight relief, the wing is much thicker at the root. However, we do not see these same trends for the Q400 and commuter cases, Figs. 6d and 6f. Instead, viscous drag has by far the largest effect on the optimal thickness-to-chord ratio. Without viscous drag, the thickness-to-chord ratio near the root is at the upper bound (we do not see this for the uCRM-9 because of wave drag). This allows the skins to be less thick due to the greater heights of the wingbox segments.

The lift values for the uCRM-9 wing, shown in Fig. 7a, are greater near the root and less near the tip as compared to an elliptical lift distribution, primarily due to the structural weight consideration in the fuel burn objective. The Q400 and commuter lift distributions are much closer to elliptical, showing that the structural weight is relatively less important in those cases, meaning the optimizer focuses more on improving aerodynamic efficiency.

Across all 21 cases, the optimal twist distributions are not greatly impacted by considering different physics. The twist distributions for the uCRM-9 and Q400 cases without viscous drag differ from the baselines slightly, as shown in Figs. $7 \mathrm{~b}$ and $7 \mathrm{~d}$. Figure $7 \mathrm{f}$ shows that without engine-weight loads, the commuter aircraft twists the outboard wing down slightly. 


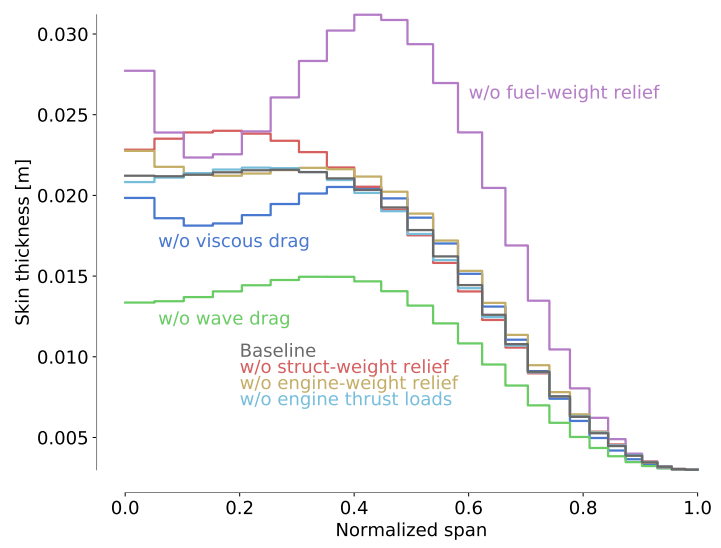

(a) uCRM-9 skin thickness distributions

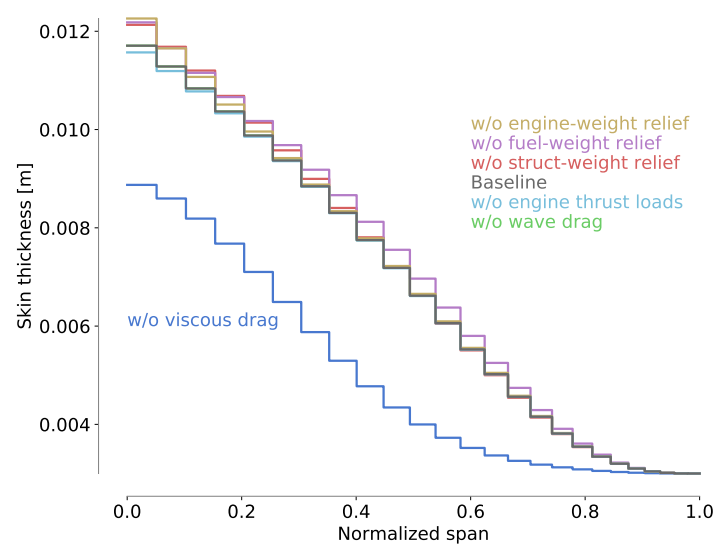

(c) Q400 skin thickness distributions

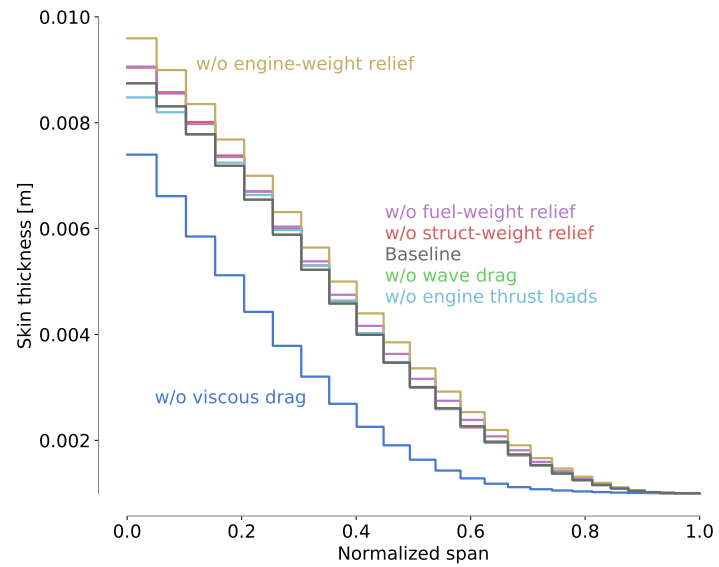

(e) Short-range commuter skin thickness distributions

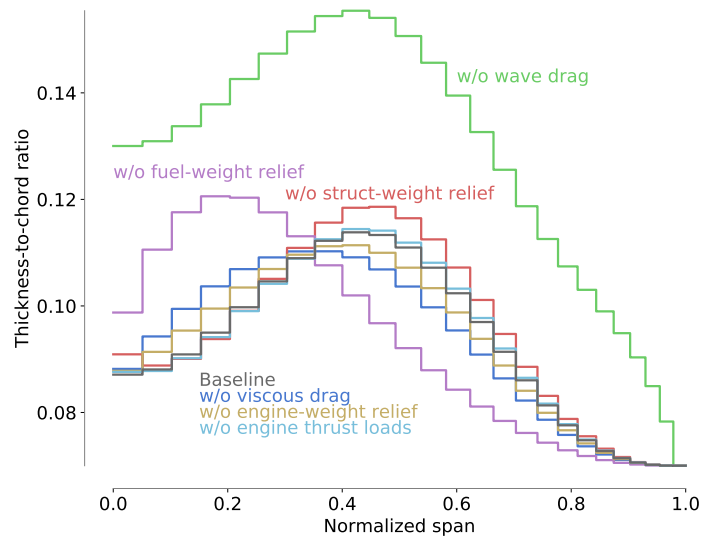

(b) uCRM-9 thickness-to-chord distributions

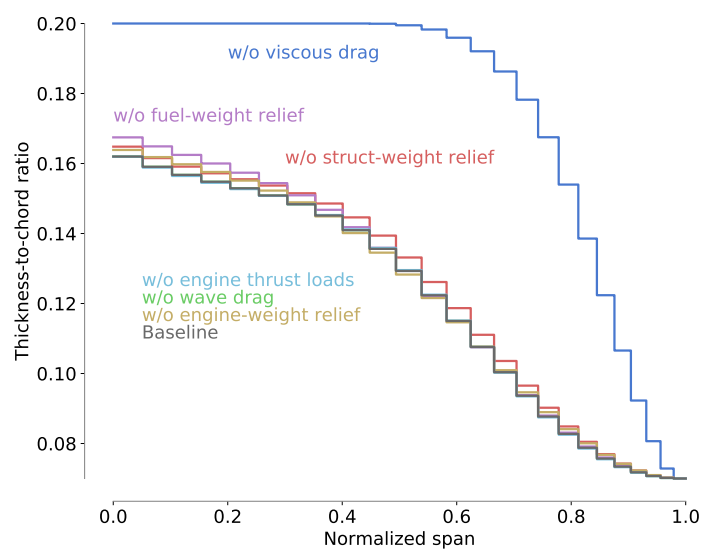

(d) Q400 thickness-to-chord distributions

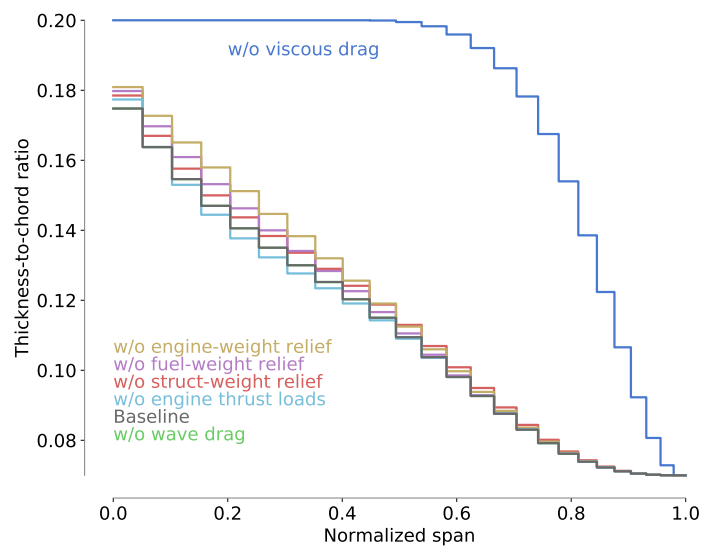

(f) Short-range commuter thickness-to-chord distributions 13

Figure 6: Optimal thickness and thickness-to-chord distributions for all of the cases for each of the three wing designs. 


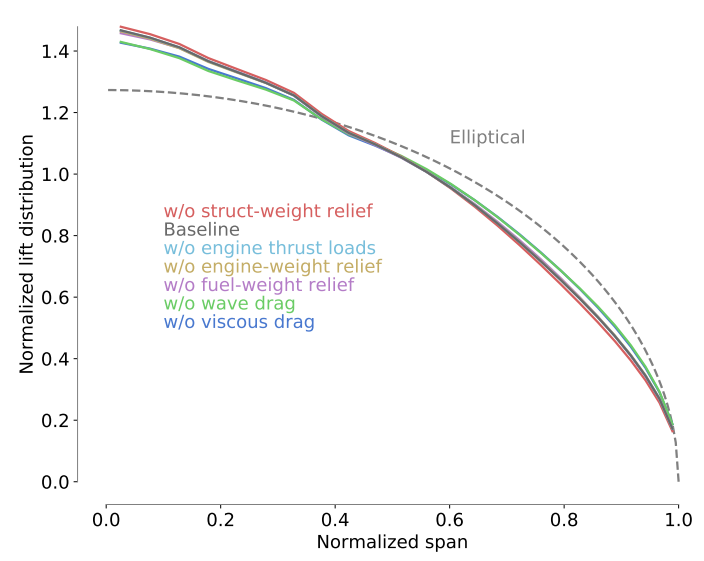

(a) uCRM-9 lift distributions

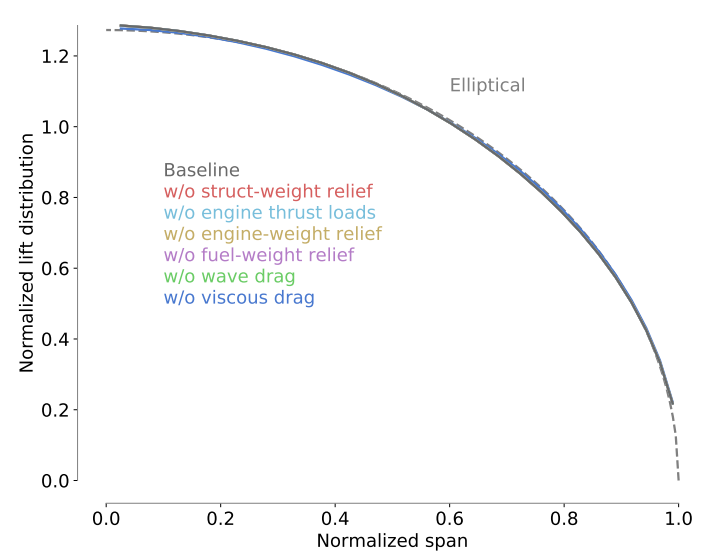

(c) Q400 lift distributions

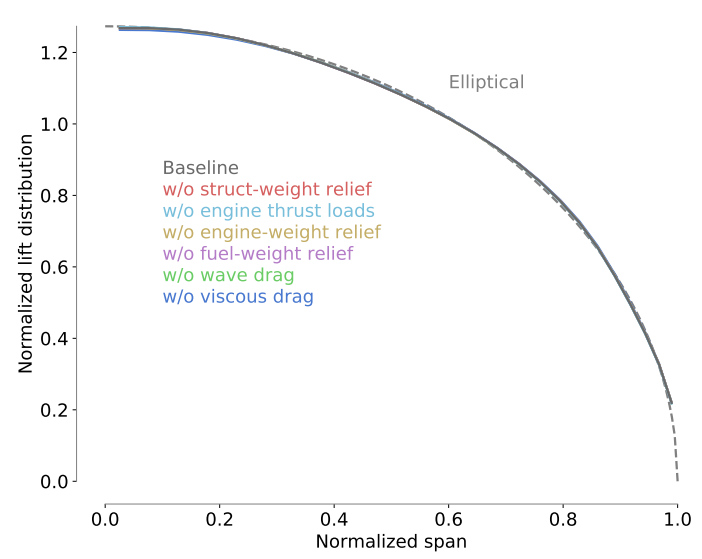

(e) Short-range commuter lift distributions

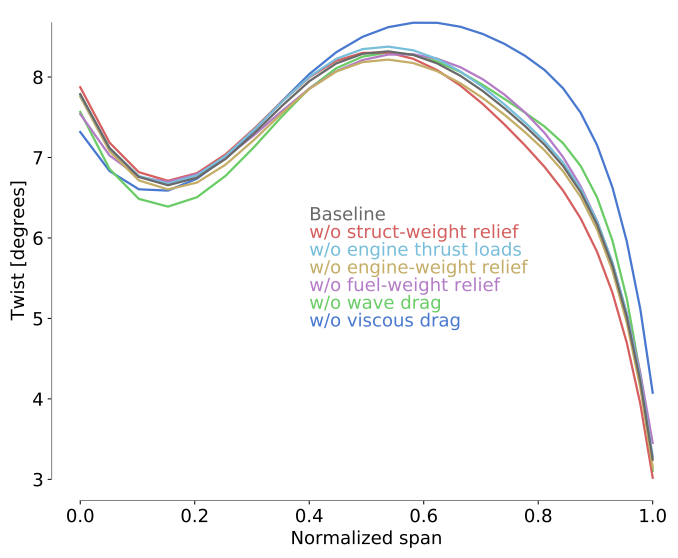

(b) uCRM-9 twist distributions

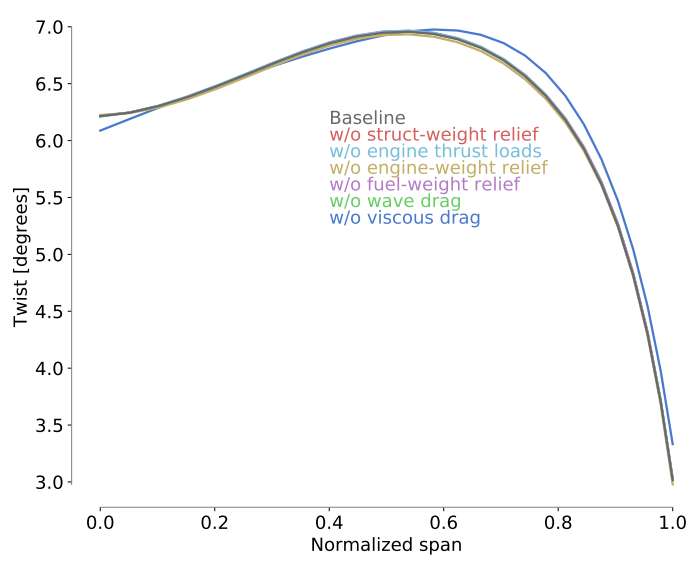

(d) Q400 twist distributions

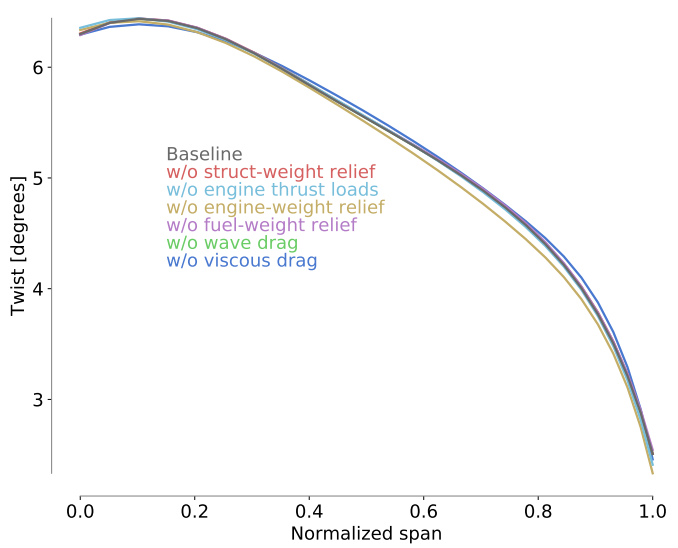

(f) Short-range commuter twist distributions

14

Figure 7: Optimal lift and twist distributions for all of the cases for each of the three wing designs. 


\section{Conclusion}

We set up and solved a series of aerostructural optimization problems for three wings of varying size (a long-range transonic-aircraft wing, a regional-aircraft wing, and a short-range commuter-aircraft wing) using the aerostructural design tool OpenAeroStruct and the modular MDO framework OpenMDAO. We started by solving a baseline optimization problem for each wing and then a series of optimization problems, each with one physical consideration removed from the baseline case, to study the relative importance of individual physical considerations on the optimal wing designs. Specifically, we looked at the effects of including viscous drag, wave drag, structural-weight load relief, fuel-weight load relief, engine-weight load relief, and engine thrust loads. We are interested in determining rough trends for each case and gaining intuition to what considerations matter most.

For the long-range transport-aircraft wing, neglecting wave drag and fuel-weight relief both changed the optimized wing mass by $30 \%$. Neglecting the structural-weight relief and viscous drag both changed the wing mass by $6 \%$. The engine-weight relief reduced the wing mass by $3 \%$ and the engine thrust had a negligible impact on the wing mass. These results indicate that for long-range transport aircraft, which usually operate in the transonic regime, ensuring that wave-drag and fuel-weight relief considerations are taken into account is important for preliminary structural sizing. Accounting for the engine-weight relief and thrust loads are the least important and can be expected to impact the wing mass by only a few percent. For the long-range transport aircraft, neglecting wave drag, structural-weight relief, fuel-weight relief, engine-weight relief, and engine thrust loads all changed the fuel burn by $5 \%$ or less, with the fuel-weight relief consideration having the greatest impact.

For the regional-aircraft wing, neglecting wave drag, structural-weight relief, fuel-weight relief, engine-weight relief, and engine thrust loads all changed the optimized wing mass by $4 \%$ or less, with the fuel-weight relief consideration having the greatest impact. Neglecting the same considerations all changed the fuel burn by less than $1 \%$. Neglecting viscous drag changed both the optimized structural mass and fuel burn by over $20 \%$. This indicates that for regional aircraft that operate in the mid-subsonic regime, ensuring that wave-drag, fuel-weight relief, structural-weight relief, engine-weight relief, and thrust-load considerations are taken into account is not important for preliminary structural sizing or fuel burn estimates. These considerations impact the wing mass and fuel burn by a few percent or less.

For the short-range commuter-aircraft wing with distributed propulsion, neglecting wave drag, structural-weight relief, fuel-weight relief, and engine thrust loads all changed the optimized wing mass by $3 \%$ or less. Neglecting engine-weight relief changed the structural mass by $9 \%$. Neglecting the same considerations all changed the fuel burn by $1 \%$ or less. Neglecting viscous drag changed both the optimized structural mass and fuel burn by over $25 \%$. This indicates that for short-range commuter aircraft with distributed propulsion that operate in the low-subsonic regime, ensuring that wave-drag, fuel-weight relief, structural-weight relief, and thrust-load considerations are taken into account is not important for preliminary structural sizing or fuel burn estimates. These considerations impact the wing mass and fuel burn by a few percent or less. However, with multiple engines distributed on the wing, engineweight relief can be expected to change the wing mass in the order of $10 \%$.

We were able to easily construct and run these optimization problems due to the flexible problem setup afforded through OpenMDAO. As model-makers, we simply define the analyses and partial derivatives for individual analysis blocks, then connect them together into a single model. OpenMDAO handles the data- and derivative-passing internally, which simplifies and expedites model creation. The modular MDO framework used here helped enable rapid and efficient exploration of the aerostructural wing design space.

Recommendations for future work include studying more physical considerations and adding more design variables to the optimization problems, such as span, sweep, and taper. Additionally, for realistic aerostructural wing design, many more load cases are required.

\section{Acknowledgements}

The first author is grateful for support from the National Science Foundation Graduate Research Fellowship under Grant No. DGE-1256260. The work presented in this paper is partially supported by NASA's Transformative Tools and Technologies (TTT) Project. 


\section{References}

[1] Maute, K., Nikbay, M., and Farhat, C., "Coupled Analytical Sensitivity Analysis and Optimization of Three-Dimensional Nonlinear Aeroelastic Systems,” AIAA Journal, Vol. 39, No. 11, 2001, pp. 2051-2061.

[2] Kenway, G. K. W., Kennedy, G. J., and Martins, J. R. R. A., "Scalable Parallel Approach for High-Fidelity Steady-State Aeroelastic Analysis and Derivative Computations," AIAA Journal, Vol. 52, No. 5, May 2014, pp. 935-951. doi:10.2514/1.J052255.

[3] Kennedy, G. J. and Martins, J. R. R. A., "A parallel aerostructural optimization framework for aircraft design studies," Structural and Multidisciplinary Optimization, Vol. 50, No. 6, December 2014, pp. 1079-1101. doi:10.1007/s00158-014-1108-9.

[4] Elham, A. and van Tooren, M. J., "Coupled adjoint aerostructural wing optimization using quasi-three-dimensional aerodynamic analysis," Structural and Multidisciplinary Optimization, Vol. 54, No. 4, 2016, pp. 889-906.

[5] Brooks, T. R., Kenway, G. K. W., and Martins, J. R. R. A., "Benchmark Aerostructural Models for the Study of Transonic Aircraft Wings," AIAA Journal, Vol. 56, No. 7, July 2018, pp. 2840-2855. doi:10.2514/1.J056603.

[6] Jasa, J. P., Hwang, J. T., and Martins, J. R. R. A., “Open-source coupled aerostructural optimization using Python,” Structural and Multidisciplinary Optimization, Vol. 57, No. 4, April 2018, pp. 1815-1827. doi:10.1007/s00158-018-1912-8.

[7] Chauhan, S. S. and Martins, J. R. R. A., "Low-Fidelity Aerostructural Optimization of Aircraft Wings with a Simplified Wingbox Model Using OpenAeroStruct," Proceedings of the 6th International Conference on Engineering Optimization, EngOpt 2018, Springer, Lisbon, Portugal, September 2018, pp. 418-431. doi:10.1007/978-3-319-97773-7_38.

[8] Alba, C., Elham, A., German, B. J., and Veldhuis, L. L., "A surrogate-based multi-disciplinary design optimization framework modeling wing-propeller interaction," Aerospace Science and Technology, Vol. 78, July 2018, pp. 721-733. doi:10.1016/j.ast.2018.05.002.

[9] Drela, M., "Method for simultaneous wing aerodynamic and structural load prediction," Journal of Aircraft, Vol. 27, No. 8, 1990, pp. 692-699.

[10] Drela, M., "Development of the D8 Transport Configuration," 29th AIAA Applied Aerodynamics Conference, American Institute of Aeronautics and Astronautics, June 2011. doi:10.2514/6.2011-3970.

[11] Ting, E., Chaparro, D., Nguyen, N., and Fujiwara, G. E., "Optimization of Variable-Camber Continuous Trailing-Edge Flap Configuration for Drag Reduction," Journal of Aircraft, Vol. 55, No. 6, 2018, pp. 2217-2239.

[12] Varello, A., Carrera, E., and Demasi, L., "Vortex lattice method coupled with advanced one-dimensional structural models," Journal of Aeroelasticity and Structural Dynamics, Vol. 2, No. 2, 2011.

[13] Brooks, T. R., Kenway, G. K. W., and Martins, J. R. R. A., "Undeflected Common Research Model (uCRM): An Aerostructural Model for the Study of High Aspect Ratio Transport Aircraft Wings," 18th AIAA/ISSMO Multidisciplinary Analysis and Optimization Conference, Denver, CO, June 2017. doi:10.2514/6.2017-4456.

[14] Gray, J. S., Hwang, J. T., Martins, J. R. R. A., Moore, K. T., and Naylor, B. A., “OpenMDAO: An open-source framework for multidisciplinary design, analysis, and optimization," Structural and Multidisciplinary Optimization, Vol. 59, No. 4, April 2019, pp. 1075-1104. doi:10.1007/s00158-019-02211-z.

[15] Salas, A. O. and Townsend, J. C., "Framework Requirements for MDO Application Development," 7th AIAA/USAF/NASA/ISSMO Symposium on Multidisciplinary Analysis and Optimization, 1998, pp. 98-4740.

[16] Padula, S. L. and Gillian, R. E., "Multidisciplinary Environments: A History of Engineering Framework Development," Proceedings of the 11th AIAA/ISSMO Multidisciplinary Analysis and Optimization Conference, Portsmouth, VA, September 2006, AIAA 2006-7083.

[17] Seider, D., Litz, M., Schreiber, A., Fischer, P. M., and Gerndt, A., "Open source software framework for applications in aeronautics and space," Aerospace Conference, 2012 IEEE, IEEE, 2012, pp. 1-11. doi:10.1109/AERO.2012.6187340.

[18] Kolonay, R. M. and Sobolewski, M., "Service ORiented Computing EnviRonment (SORCER) for Large Scale, Distributed, Dynamic Fidelity Aeroelastic Analysis," Optimization, International Forum on Aeroelasticity and Structural Dynamics, IFASD 2011, 26-30, 2011.

[19] Makhija, D. and Beran, P. S., "Spiral: A general framework for parameter sensitivity analysis," 58th AIAA/ASCE/AHS/ASC Structures, Structural Dynamics, and Materials Conference, 2017, p. 1306.

[20] Hwang, J. T. and Martins, J. R. R. A., "A computational architecture for coupling heterogeneous numerical models and computing coupled derivatives," ACM Transactions on Mathematical Software, Vol. 44, No. 4, June 2018, pp. Article 37. doi:10.1145/3182393.

[21] Ning, A. and Petch, D., "Integrated Design of Downwind Land-based Wind Turbines using Analytic Gradients," Wind Energy, Feb. 2016, pp. 1-17. doi:10.1002/we.1972. 
[22] Stanley, A. P. J., Ning, A., and Dykes, K., "Coupled Wind Turbine Design and Layout Optimization with Non-Homogeneous Wind Turbines," Wind Energy Science, July 2018. doi:10.5194/wes-2018-54.

[23] Gray, J. S., Mader, C. A., Kenway, G. K. W., and Martins, J. R. R. A., "Modeling Boundary Layer Ingestion Using a Coupled Aeropropulsive Analysis," Journal of Aircraft, Vol. 55, No. 3, May 2018, pp. 1191-1199. doi:10.2514/1.C034601.

[24] Gray, J. S. and Martins, J. R. R. A., "Coupled Aeropropulsive Design Optimization of a Boundary Layer Ingestion Propulsor," The Aeronautical Journal, Vol. 123, No. 1259, January 2019, pp. 121-137. doi:10.1017/aer.2018.120.

[25] Jasa, J. P., Gray, J. S., Seidel, J. A., Mader, C. A., and Martins, J. R. R. A., "Multipoint Variable Cycle Engine Design Using Gradient-based Optimization," 57th AIAA Aerospace Sciences Meeting, AIAA SciTech Forum, 2019, San Diego, CA, January 2019. doi:10.2514/6.2019-0172.

[26] Hendricks, E. S., Falck, R. D., and Gray, J. S., "Simultaneous Propulsion System and Trajectory Optimization," 18th AIAA/ISSMO Multidisciplinary Analysis and Optimization Conference, Denver, CO, June 2017.

[27] Falck, R. D., Chin, J. C., Schnulo, S. L., Burt, J. M., and Gray, J. S., “Trajectory Optimization of Electric Aircraft Subject to Subsystem Thermal Constraints," 18th AIAA/ISSMO Multidisciplinary Analysis and Optimization Conference, Denver, CO, June 2017.

[28] Martins, J. R. R. A., Sturdza, P., and Alonso, J. J., "The Complex-Step Derivative Approximation," ACM Transactions on Mathematical Software, Vol. 29, No. 3, September 2003, pp. 245-262. doi:10.1145/838250.838251.

[29] Jasa, J. P., Hwang, J. T., and Martins, J. R. R. A., "Design and Trajectory Optimization of a Morphing Wing Aircraft," 2018 AIAA/ASCE/AHS/ASC Structures, Structural Dynamics, and Materials Conference; AIAA SciTech Forum, Orlando, FL, January 2018. doi:10.2514/6.2018-1382.

[30] Chaudhuri, A., Jasa, J., Martins, J. R. R. A., and Willcox, K., "Multifidelity Optimization Under Uncertainty for a Tailless Aircraft," 2018 AIAA/ASCE/AHS/ASC Structures, Structural Dynamics, and Materials Conference; AIAA SciTech Forum, Orlando, FL, January 2018. doi:10.2514/6.2018-1658.

[31] Hwang, J. T. and Ning, A., "Large-scale multidisciplinary optimization of an electric aircraft for on-demand mobility," 2018 AIAA/ASCE/AHS/ASC Structures, Structural Dynamics, and Materials Conference, Kissimmee, FL, January 2018. doi:10.2514/6.2018-1384.

[32] Lambe, A. B. and Martins, J. R. R. A., "Extensions to the Design Structure Matrix for the Description of Multidisciplinary Design, Analysis, and Optimization Processes," Structural and Multidisciplinary Optimization, Vol. 46, August 2012, pp. 273284. doi:10.1007/s00158-012-0763-y.

[33] Raymer, D. P., Aircraft Design: A Conceptual Approach, AIAA, 5th ed., 2012.

[34] Bons, N. P., He, X., Mader, C. A., and Martins, J. R. R. A., "Multimodality in Aerodynamic Wing Design Optimization," 18th AIAA/ISSMO Multidisciplinary Analysis and Optimization Conference, Denver, CO, June 2017. doi:10.2514/6.2017-3753.

[35] Bons, N., He, X., Mader, C. A., and Martins, J. R. R. A., "Multimodality in Aerodynamic Wing Design Optimization," AIAA Journal, Vol. 57, No. 3, March 2019, pp. 1004-1018. doi:10.2514/1.J057294.

[36] Malone, B. and Mason, W., "Multidisciplinary Optimization in Aircraft Design Using Analytic Technology Models," Journal of Aircraft, Vol. 32, No. 2, mar-apr 1995, pp. 431-438. doi:10.2514/3.46734.

[37] Johnson, W., Silva, C., and Solis, E., “Concept Vehicles for VTOL Air Taxi Operations,” 2018.

[38] Vassberg, J. C., DeHaan, M. A., Rivers, S. M., and Wahls, R. A., "Development of a Common Research Model for Applied CFD Validation Studies,” 2008. doi:10.2514/6.2008-6919, AIAA 2008-6919.

[39] Chauhan, S. S., Hwang, J. T., and Martins, J. R. R. A., "An automated selection algorithm for nonlinear solvers in MDO," Structural and Multidisciplinary Optimization, Vol. 58, No. 2, June 2018, pp. 349-377. doi:10.1007/s00158-018-2004-5.

[40] Jameson, A., Leoviriyakit, K., and Shankaran, S., "Multi-point aero-structural optimization of wings including planform variations," 45th AIAA Aerospace Sciences Meeting and Exhibit, 2007, p. 764.

[41] Hürlimann, F., Kelm, R., Dugas, M., Oltmann, K., and Kress, G., "Mass estimation of transport aircraft wingbox structures with a CAD/CAE-based multidisciplinary process," Aerospace Science and Technology, Vol. 15, No. 4, 2011, pp. $323-333$.

[42] Gill, P. E., Murray, W., and Saunders, M. A., "SNOPT: An SQP Algorithm for Large-Scale Constrained Optimization,” SIAM Review, Vol. 47, No. 1, 2005, pp. 99-131. doi:10.1137/S0036144504446096.

[43] Perez, R. E., Jansen, P. W., and Martins, J. R. R. A., “pyOpt: A Python-Based Object-Oriented Framework for Nonlinear Constrained Optimization,” Structural and Multidisciplinary Optimization, Vol. 45, No. 1, January 2012, pp. 101-118. doi:10.1007/s00158-011-0666-3.

[44] Kraft, D., “A software package for sequential quadratic programming,” Tech. rep., Tech. Rep. DFVLR-FB 88-28, DLR German Aerospace Center, 1988. 